\title{
Chaos, cheating and cooperation: potential solutions to the Prisoner's Dilemma
}

\author{
Björn Brembs
}

Brembs, B. 1996. Chaos, cheating and cooperation: potential solutions to the Prisoner's Dilemma. - Oikos 76: 14-24

The Iterated Prisoner's Dilemma (IPD) is widely regarded as a standard model for the evolution of cooperation. This review tries to give an outline of the development in the field since Axelrod and Hamilton (1981) spawned an avalanche of papers with the announcement of Tit-for-tat as the winner of their computer tournament. The most important advancements in the game-theoretical work on different aspects of the game are described. It becomes evident that changing any of the numerous parameters of the game will inevitably change the outcome; there is virtually no end to the IPD. Using experimental data from various taxa, the applicability of the IPD in nature is analysed and potential future developments in the area are discussed.

B. Brembs, Theodor-Boveri-Institut für Biowissenschaften (Biozentrum), Dept of Genetics, Am Hubland, D-97074 Würzburg, Germany.

\section{The game}

"No instinct has been produced for the exclusive good of other animals, but each animal takes advantage of the instincts of others" (Darwin 1859). Ever since Darwin the evolutionary success of a seemingly obvious contradiction to his statement has raised the interests of naturalists all over the world: some cooperating animals are clearly mutualistic or even altruistic. Before the 1960s only a few scientists attempted to understand the evolutionary processes underlying cooperation. since group selection seemed to explain cooperative societies. Yet. research in later years could not support a pervasive group-benefit view of selection; how then can cooperative genotypes spread in an environment of selfish genes? Currently, the evolution of cooperation can be divided into several general categories: e.g. 1) by-product mutualism where cooperation is an incidental outcome from genuinely selfish behaviour (Dugatkin et al. 1992) kin-selected altruism (Hamilton 1964) with its climax in the social insects and 3) reciprocal altruism (Trivers 1971) among unrelated individuals. The 'Prisoner's Dilemma' is used as the standard metaphor to conceptualise the conflict between mutual support and selfish exploitation among interacting nonrelatives in biological communities. It has yielded a plethora of investigations concerned with a theory of cooperation based on reciprocal altruism. The

Accepted 31 August 1995

Copyright (c) OIKOS 1996

ISSN 0030-1299

Printed in Ireland - all rights reserved 
Prisoner's Dilemma is a simple two-person game, where each player (for instance two prisoners accused of the same crime) can choose either to cooperate $(\mathrm{C})$ or to defect $(\mathrm{D}=$ not cooperate). Fig. 1 shows the payoff matrix: if one player defects, the other has the option to cooperate yielding $\mathrm{S}$ (the sucker's payoff or to defect and obtain $\mathrm{P}$ (the punishment for mutual defection). On the other hand. if the opponent cooperates, one receives $\mathrm{R}$ (the reward for cooperation) for a $\mathrm{C}$ or gains $\mathrm{T}$ (the temptation to defect) for a D. Provided the payoffs satisfy

$\mathrm{T}>\mathrm{R}>\mathrm{P}>\mathrm{S}$, with $\mathrm{R}>(\mathrm{S}+\mathrm{T}) / 2,(1)$

and the players meet only once, each player should defect no matter what the adversary does, in order not to become the 'sucker'. In a population of interacting pairs of individuals as described above, no single mutant adopting a different strategy can invade and secure a foothold. Defection is the primeval state and the only evolutionary stable strategy (ESS; Maynard Smith 1982). However, when played repeatedly, there is no trivial answer to the question of how cooperation can arise from an non-cooperative state.

\section{Deterministic strategies}

Let there be a homogenous population with individuals that are able to recognise a previous interactant and remember some aspects of prior outcomes. If the average chance to meet a given partner more than $\Theta$ times in a row is $w^{\Theta}$ (where $0<w<1$ ), then a strategy would be a rule, modifying the behaviour (C or D) according to the history of the interaction so far. Obviously, unconditional defection (ALLD) is still an ESS against single cooperating mutants. But there may be other stable strategies as well. In fact, in the Iterated Prisoner's Dilemma (IPD) no strategy is evolutionarily stable (Boyd and Lorberbaum 1987, Lorberbaum 1994).

\section{Tit for tat}

Axelrod and Hamilton (1981) used a computer

\begin{tabular}{ll|l|l}
\multicolumn{4}{c}{ Player 2 } \\
& & Cooperate & Defect \\
\cline { 2 - 4 } & Cooperate & $\mathrm{R}=3$ & $\mathrm{~S}=0$ \\
\hline Defect & $\mathrm{T}=5$ & $\mathrm{P}=1$
\end{tabular}

Fig. 1. The payoff matrix of the Prisoner's Dilemma: The two participants have two options: to cooperate or to defect. The payoff to player one is shown. Obviously, he gains more by defection not only if player two cooperates (5 instead of 3 points), but also if player two defects ( 1 instead of 0 points). Since this is also valid for player two, both end up defecting and score instead of 3 points each if they had cooperated. Hence the dilemma. tournament to detect strategies that would favour cooperation among individuals engaged in the IPD. In a first round, 14 more or less sophisticated strategies and one totally random strategy competed against each other for the highest average scores in an IPD of 200 moves. Unexpectedly, a very simple strategy did outstandingly well: cooperate on the first move and then copy your opponent's last move for all subsequent moves. This strategy was called 'Tit for tat' (TFT) and became the founder of an ever growing amount of successful strategies. In a similar competition with 62 contestants, TFT won again. It has three characteristics that account for its impressive performance: it is nice (cooperates on the first move), retaliatory (punishes defection in the prior move with defection) and forgiving (immediate return to cooperation after one $\mathrm{C}$ of the adversary). In an ecological analysis, the scores from round two were used to calculate the relative frequencies of the strategies in a hypothetical population. The strategies were then submitted to each subsequent round in proportion to their cumulative payoff $(\boldsymbol{V})$ in the previous round. In the long run, TFT outcompeted its rivals and went to fixation (Axelrod and Hamilton 1981). Moreover, for

$w \geq \max \left[\frac{T-R}{T-P}\right] ;\left[\frac{T-R}{R-S}\right]$

where max denotes the larger of the two values in brackets, TFT can increase in frequency in a predominantly ALLD environment and, once established, resists invasion of any defecting strategy. Under these circumstances, no single mutant can do better than TFT (TFT is "robust", Axelrod and Hamilton 1981). Notwithstanding, there may be "twin"-strategies, that do as well as TFT against TFTers. Any other cooperating strategy (e.g. "always cooperate", ALLC) can perform a 'random walk' through a homogenous TFT-population as they both get the same reward $\mathrm{R}$. The relative fitness of both strategies is equal, thus the selection coefficient $\mathrm{s}=0$.

\section{TF2T and STFT}

Moreover, Boyd and Lorberbaum (1987) showed that no deterministic (pure) strategy is evolutionarily stable in the IPD. Consider the following line of argument: a strategy $S_{e}$ is robust (or "collectively stable", Boyd and Lorberbaum 1987) if for any given strategy $S_{\mathrm{i}}$ :

$\mathrm{V}\left(\mathrm{S}_{\mathrm{e}} \mid \mathrm{S}_{\mathrm{e}}\right)>=\mathrm{V}\left(\mathrm{S}_{\mathrm{i}} \mid \mathrm{S}_{\mathrm{e}}\right)$

where $\mathrm{V}\left(\mathrm{S}_{\mathrm{m}} \mid \mathrm{S}_{\mathrm{n}}\right)$ is the payoff of individuals using strategy $\mathrm{Sm}$ when interacting with individuals using strategy $S_{n}$. Consider a randomly meeting population, consisting of $\mathrm{n}$ strategies with the 
frequencies $f_{1}, f_{2}, \ldots, f_{n}$. Furthermore, a common strategy $S_{e}$ coexists with n-1 rare strategies, so that $f_{e} \gg f_{j}$ (e unequal $j$ ). In order to fulfil this assumption, the inequality

$$
\sum_{j=1}^{n} f_{j} V\left(S_{e} \mid S_{j}\right)>\sum_{j=1}^{n} f_{j} V\left(S_{i} \mid S_{j}\right)
$$

needs to be satisfied. This is still the case, if for some $\mathrm{i}, \mathrm{V}\left(\mathrm{S}_{\mathrm{e}} \mid \mathrm{S}_{\mathrm{e}}\right)=\mathrm{V}\left(\mathrm{S}_{\mathrm{i}} \mid \mathrm{S}_{\mathrm{e}}\right)$. There may be even strategies $\mathrm{S}_{\mathrm{i}}$, for which $\mathrm{V}\left(\mathrm{S}_{\mathrm{i}} \mathrm{S}_{\mathrm{i}}\right)=\mathrm{V}\left(\mathrm{S}_{\mathrm{e}} \mid \mathrm{S}_{\mathrm{i}}\right)$ and (4) still is true. For example, the nice strategy TFT has the same expected fitness as another nice strategy Tit for two tats (TF2T, allowing two consecutive Ds before retaliating) when interacting, because neither ever defects. The relative fitness of TFT and TF2T is dependent upon their interaction with any third strategy. Assume this third strategy is Suspicious tit for tat (STFT, playing D in the first interaction), maintained at a low level in the population through recurrent mutation (for example). For $\mathrm{V}(\mathrm{TF} 2 \mathrm{~T} \mid \mathrm{STFT})>\mathrm{V}(\mathrm{TFT} \mid \mathrm{STFT})$ TF2T can invade TFT without random drift. If (2) holds, this is always true because the more tolerant TF2T invokes cooperation in STFT, whereas the retaliatory TFT defects together with STFT. Accordingly, any deterministic strategy can be invaded and outcompeted by the joint effect of a "twin"-strategy together with another strategy. Boyd and Lorberbaum (1987) proved this general case to be true for all $\boldsymbol{w}>\min [(\mathrm{T}-\mathrm{R}) /(\mathrm{T}-\mathrm{P}), \quad(\mathrm{P}-\mathrm{S}) /(\mathrm{R}-\mathrm{S})]$. Lorberbaum (1994) extended the instability proof to stochastic (mixed) strategies as well. Depending on the initial set of assumptions, however, there are different types of evolutionary stability of single strategies (Bender and Swistak 1995) or of groups of strategies (see below) in the IPD. All of which have to be considered before seriously testing the model.

\section{Free Rider and Rover}

Soon after these findings, it became clear that a 'mobile individual using an ALLD strategy could exploit a population of cooperators, simply by switching to new partners (i.e. manipulating $\boldsymbol{w}$ for its own purposes) when the sucker ceased to cooperate (Dugatkin and Wilson 1991, Enquist and Leimar 1993). There are three components modulating the expected frequencies of cooperation: 1) If search time $\tau$ is low, individuals adopting this strategy are favoured. 2) The same is true, if the population is large enough for new, 'naive' individuals to be easily encountered. 3) The average coalition duration $\Theta$ modifies the availability of potential 'victims': a decrease in $\Theta$ will result in an increase in splitting of cooperating couples which leads to single individuals looking for new potential partners. In order to determine the extent to which these factors influence cooperation, Enquist and Leimar (1993)

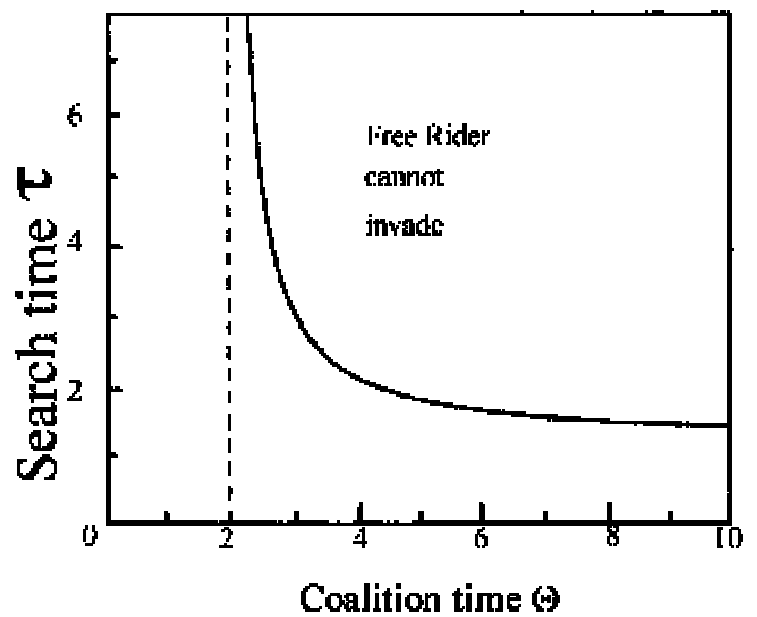

Fig. 2. For certain combinations of coalition time and search time, a population of cooperating individuals cannot be invaded by FR. The dashed line denotes the minimum average coalition time for an IPD with $\mathrm{T}=2, \mathrm{R}=1, \mathrm{P}=0$ and $\mathrm{S}=-1$. Redrawn from: Enquist, M. and Leimar, 0. 1993. The evolution of cooperation in mobile organisms. Anim. Behav. 45: 749.

analysed a model in which "Free Riders" (FR), an ALLD strategy that abandons defecting partners, occur. Analogous to (3), the stability condition for TFT against a FR is $\mathrm{V}(\mathrm{TFT} \mid \mathrm{TFT})>\mathrm{V}(\mathrm{FR} \mid \mathrm{TFT})$.

In an essentially infinite population, the search time $\tau$ is assumed to be exponentially distributed (Enquist and Leimar 1993). Fig. 2 shows the relations between $\tau$ and $\Theta$ for which a cooperating population is stable against a FR. For long search times the stability condition approaches $\Theta>\mathrm{T} / \mathrm{R}$. For shorter $\tau$ the coalition time has to be even longer: $\Theta>\tau T(\tau R+S)$ and for $\tau \leq-S / R$ cooperation will never be evolutionarily stable. (Enquist and Leimar 1993; they used a different terminology. In their payoff matrix $\mathrm{P}=0$ and $\mathrm{S}<0$ ). Similar results were obtained from a slightly more intricate model by Dugatkin and Wilson (1991). Their strategy 'Rover' was exploiting cooperators in a patchy environment. As long as $\tau_{1}>\tau>\tau_{2}$, they found Rover/TFT dimorphisms to be stable as well. Here $\tau_{1}$ is the upper threshold above which TFT is evolutionarily stable against Rover and $\tau_{2}$ the lower threshold, below which Rover sweeps to fixation (see also Feldmann and Thomas 1987 for further conditions for polymorphism stability).

\section{Escaping the IPD}

In order to avoid exploitation by travelling defectors, one can imagine several countermoves: while xenophobia or initiation rites may increase travel cooperating individual may also try the round: decrease each investment in cooperation and compensate by increasing the number of interactions, until the net gain of defection will drop below $\tau$. As a result, average coalition time $\Theta$ would 
be prolonged and the system may come to lie above the line, depicted in Fig. 2.

In Axelrod's original definition, this would correspond to $\mathrm{R}>\mathrm{T}$, which means that selfishness and altruism become equivalent. Such a strategy would have put itself outside the IPD, manipulating the payoff matrix. If that rule also altered $S>P$, defection would never pay and ALLC would be the sole ESS (Mesterton-Gibbons 1991). The resulting cooperative system would certainly be classified as by-product mutualism, according to the definition by Dugatkin et al. (1992).

\section{Observer TFT}

As another possibility, gathering information about a future interactant could be efficient as well: observation of a defecting individual would result in playing D when this individual is encountered, reducing the overall payoff to the defector. Consider the strategy Observer tit for tat (OTFT): it is identical to TFT when ignorant of a new partner, but starts by defecting, if this partner was seen defecting on another (Pollock and Dugatkin 1992). On what conditions does OTFT outperform TFT? Remarkably, this only occurs when $\boldsymbol{w}$ is relatively small and the proportion of defectors is sufficiently high. The results of Pollock and Dugatkin (1992) suggest that OTFT only in part compensates for the low value of $\boldsymbol{w}$, reducing ALLD fitness by diminishing the number of suckers: in a Rover/TFT polymorphism, OTFT is not able to extinguish Rover, it only decreases its frequency (Pollock and Dugatkin 1992). The described features reflect the insight that social controls will only be maintained if exploitation actually poses a severe threat. As the probability of future interaction increases, the chance of punishing defectors increases, rendering third-party observation superfluous.

\section{Stochastic strategies}

The last section already hinted at the vast number of difficulties facing cooperation in a selfishly exploitative environment if it still satisfies the conditions of an IPD. It appears even more difficult, if the individuals operate in a 'noisy' environment. Certain actions may be misinterpreted due to random malfunctions in perception or transmission and retaliatory individuals such as TFTers end up in a sequence of reprisals until the next error occurs.

\section{Generous TFT}

According to Nowak and Sigmund (1992, 1993a, b) this weakness is caused by the deterministic nature of the strategies discussed so far. In reducing the probability for $\mathrm{C}$ or $\mathrm{D}$ specified by the outcome of the previous round from 1 to $<1$, one takes random errors into account. For the infinitely IPD (the limit case, $\boldsymbol{w}=1$ ) the first move is irrelevant since its effect is 'forgotten' in the long run (Nowak and Sigmund 1992). The decision rule then consists of a point ( $p$, q) in the unit square, with the probability p (q) for a $\mathrm{C}$ after a cooperative (defective) move of the partner; $0<\mathrm{p}, \mathrm{q}<1$. In this terminology TFT corresponds to $(1,0), \operatorname{ALLD}$ to $(0,0), \operatorname{ALLC}$ to $(1,1)$ and so on. In such a scenario, Generous tit for tat (GTFT), a more forgiving strategy with $\mathrm{q}=\min [1-(\mathrm{T}-$ $\mathrm{R}) /(\mathrm{R}-\mathrm{S}),(\mathrm{R}-\mathrm{P}) /(\mathrm{T}-\mathrm{P})]=1 / 3$ is said to provide the highest payoff (Nowak and Sigmund 1992).

Nowak and Sigmund (1992) used a similar approach as Axelrod in his ecological tournament: 100 strategies $S_{1}$ to $S_{100}$ (uniformly distributed on the unit square, equal initial relative frequencies) were sampled, frequencies were assessed according to the payoff of the previous round, below-threshold strategies were discarded and the frequencies evolved as Fig. 3 shows: initially, the strategies closest to $\operatorname{ALLD}(0,0)$ feed on a large percentage of high q suckers, and increase drastically while the others vanish. If some of the initial strategies are close to TFT $(1,0)$, however, the strategies near ALLD will never reach fixation: the depletion of suckers reduces the exploiters' fitness (they now gain mostly $\mathrm{P}$ ) while the TFT-like strategies still obtain $\mathrm{R}$ interacting among themselves and $\mathrm{P}$ confronting the defecting strategies. Slowly at first but gathering momentum, the cooperators increase in frequency and the defectors wane. Yet, having extinguished the ALLD-like strategies, the rules close to TFT are superseded by a third strategy, very close to the less severe retaliator GTFT. Due to the noise, generated by the stochasticity of the decision rules, the harsh retaliation of TFT proved 'fatal' to TFT. The possibility of forgiveness invading a retaliatorily cooperating community has previously been shown (Boyd and Lorberbaum 1987); however, the simulations conducted by Nowak and Sigmund (1992) clearly demonstrate the 'catalyser'-effect of TFT:

\footnotetext{
"TFT's strictness is salutary for the community, but harms its own. [...] It needs to be present, initially only in a tiny amount; in the intermediate phase, its concentration is high; but in the end, only a trace remains."
}

\section{Pavlov}

Extending their simulations (Nowak and Sigmund 1993a) to more complex strategies and taking not only the opponent's last move but also one's own into account, the decision rule is given by a fourdimensional vector $\left(\mathrm{p}_{1}, \mathrm{p}_{2}, \mathrm{p}_{3}, \mathrm{p}_{4}\right)$ for cooperating after R, S, T and P. [e.g. TFT: (1, 0, 1, 0); Grim: (1, $0,0,0)$ after a single $\mathrm{D}$ of the opponent never revert to $\mathrm{C}$ again].

Each of the 40 simulations was started with the random strategy $(0.5,0.5,0.5,0.5)$ and at every 100th generation (on average) a small amount of one 


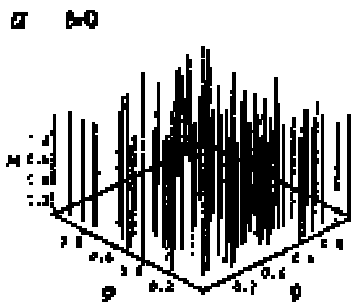

tat

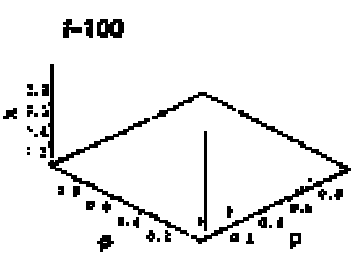

$+200$

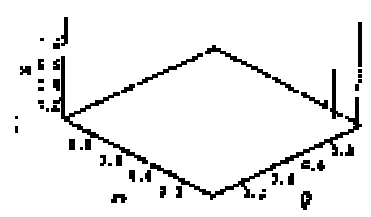

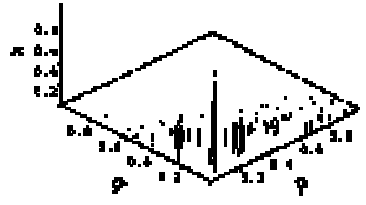

In1내

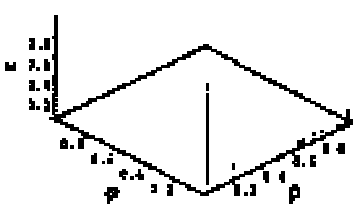

$\rightarrow 1,000$

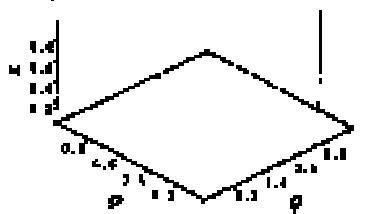

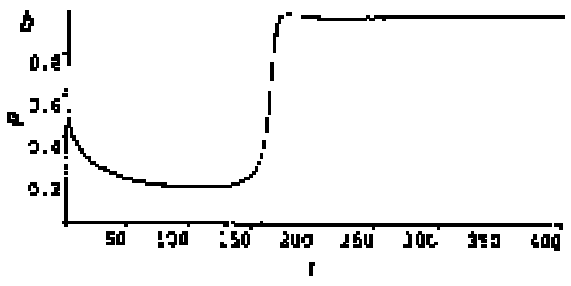
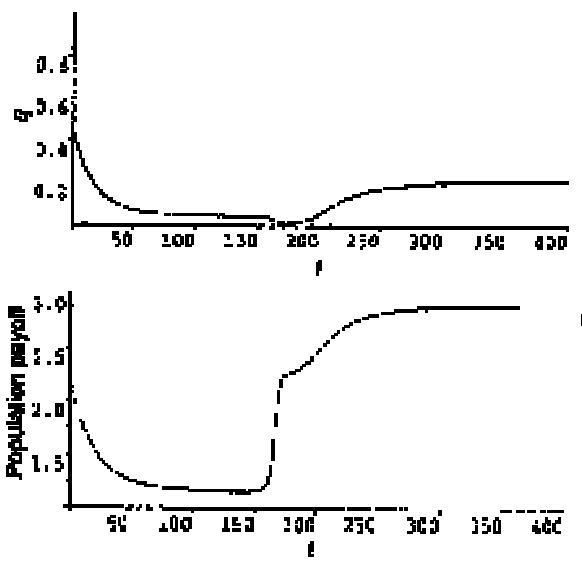

Fig. 3. The evolution of a set of strategies is shown. 99 strategies were randomly sampled and a TFT-like $(0.99,0.01)$ strategy was added. The initial frequencies $\mathrm{f}$ were uniformly set to 0.01 and all subsequent frequencies evolved in proportion to the payoff of the previous round. a) Relative frequencies after $0,20,100,150,200,1000$ generations. Strategies with very low frequencies may disappear from this plot, although they are present in the numerical computations. b) Population averages for p, q and payoff. See text for details. From: Nowak, M. A. and Sigmund, K. 1992. Tit for tat in heterogeneous populations. Nature 355: 251.

of $10^{5}$ randomly chosen different mutant strategies was introduced. Mutations were limited to $\mathrm{p}_{1}-\mathrm{p}_{4}$; all other parameters (i.e. $\boldsymbol{w}, \mathrm{R}, \mathrm{S}, \mathrm{T}, \mathrm{P}$, etc.) were fixed. The frequencies evolved according to the rules described above. Fig. 4 shows a scenario that is similar to that of the Punctuated equilibrium (Eldredge and Gould 1972): rapid shifts in the beginning, followed by an increase of defective strategies, replaced by a short phase of stable TFTlike dominance and finally substituted by GTFT or, in more than $80 \%$, of the simulations, by a new strategy. The newcomer was close to $(1,0,0,1)$ : cooperate after $\mathrm{R}$ and $\mathrm{P}$, defect after $\mathrm{S}$ and $\mathrm{T}-$ in other words, stay with the previous decision after scoring the higher payoffs $\mathrm{R}$ and $\mathrm{T}$ and switch after $\mathrm{S}$ and $\mathrm{P}$.

Because of its reflexive nature, Nowak and Sigmund dubbed it "Pavlov", but corresponding rather to operant than to classical conditioning, it should reasonably have been called "Skinner", for example. Anyway, Pavlov's advantage over TFT is based on two important features: it can correct occasional mistakes and prevents invasion of strict cooperators by exploiting them. In contrast to TFT, Pavlov loses against ALLD, because it alternates between $\mathrm{C}$ and D. By changing the decision rule slightly (e.g. 0.999,
0.001, 0.001, 0.995), however, this Pavlov-like strategy is evolutionarily stable against ALLD.

\section{A different solution: chaos}

\section{Frequential chaos}

If Gould and Eldredge's (1993) statement that "maintenance of stability within species must be considered as a major evolutionary problem" is also valid for cornpeting strategies (i.e. genotypes) in the IPD, the findings of Nowak and Sigmund (1993b) deserve special interest. According to their terminology, there is a set of 16 deterministic strategies $S_{0}$ to $S_{15}$, representing the 16 corner-points of the four-dimensional vector space (i.e. $\mathrm{S}_{0}$ is ALLD, $S_{9}$ is Pavlov, $S_{10}$ is TFT, etc.). As in the last simulation, noise is taken into account by replacing 1 by 1 -e and 0 by e in the vectors; with $0<\mathrm{e}<<1$, the first round no longer matters (Nowak and Sigmund 1993b). 

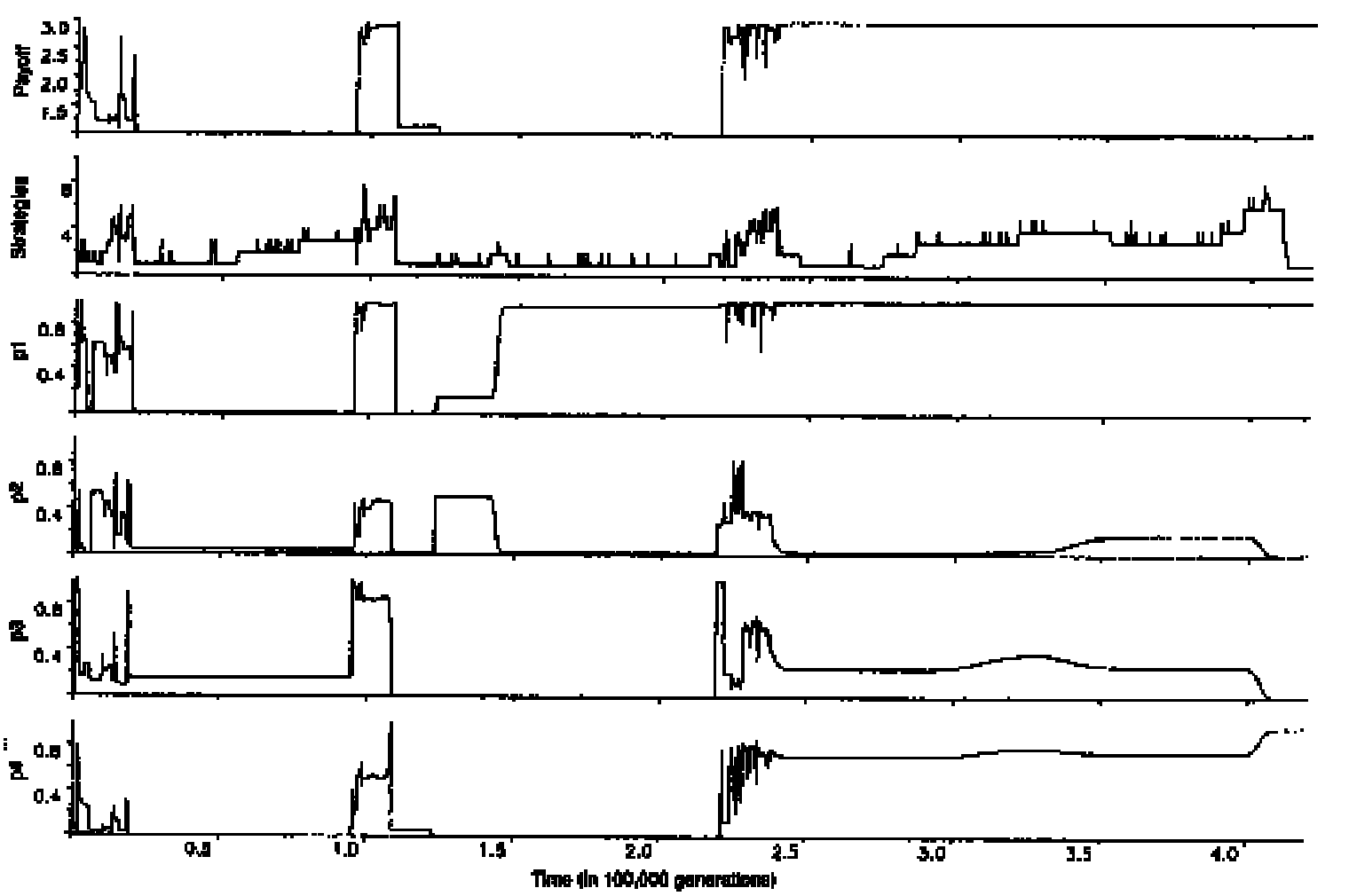

Fig. 4. An evolutionary simulation of strategies involved in the IPD. The simulation was opened with the random strategy $(0.5,0.5,0.5,0.5)$. In each generation there is a 0.01 probability of mutation (i.e. randomising $\mathrm{p}_{1}$ and $\mathrm{p}_{2}$ ). The relative frequencies were distributed according to the payoffs in the previous generation. Strategies with frequencies below 0.001 were discarded. Here, violent initial shifts are followed by the dominance of an ALLD-like mutant. At $t=92000$ a TFT-like strategy invades and gets overrun by GTFT. As more forgiving strategies drift into the cooperative society, defective strategies are able to invade and defection, dominated by Grim $(0.999,0.001,0.001,0.001)$ is the rule. Again TFT invades and is superseded, this time by the Pavlov-like $(0.999,0.001,0.007,0.946)$. This persists until $\mathrm{t}=107$ (not shown). The figure shows the average population payoff. the total number of strategies and the population averages of $\mathrm{p} 1$ through $\mathrm{p} 4$. See text for more details. From: Nowak, M. A. and Sigmund, K. 1993. A strategy of win-stay lose-shift that outperforms tit-fortat in the prisoner's dilemma game. Nature 364: 57.

Nowak and Sigmund computed a large population consisting of $S_{0}$ to $S_{15}, f_{i}$ being the relative frequency of $S_{i}$ in a given generation, $V_{i}$ the average payoff for an $S_{i}$ player and $\boldsymbol{u}$ a tiny number of invaders. Selection was computed as described above. This yields

$f_{i}^{\prime}=\frac{\left(\frac{f_{i} V_{i}}{\sum f_{i} V_{i}}+u\right)}{1+\mathrm{n} u}, \mathrm{i}=1, \ldots, \mathrm{n}$

where $f_{i}$ ' denotes the frequency of $S_{i}$ after one generation. Eq. (5) exhibits complex dynamics. The frequencies may oscillate periodically or display violently chaotic orbits. For example, around $\boldsymbol{u}=$ $0.0004, S_{4}, S_{6}, S_{7}$ and $S_{12}$ almost perish, while the other strategies oscillate vigorously. Only TFT persists at comparatively high frequency, whereas the other oscillating strategies come very close to $f_{i}=0$. But whenever $f_{10}$ (TFT) is high, it is outcompeted by a more generous strategy $S_{11}(1,0$, $1,1)$ and $S_{9}$ (Pavlov), which in turn are invaded by the parasitic $S_{1}(0,0,0, I) . S_{1}$ is then sacked by ALLD-like $\left(\mathrm{S}_{0}\right)$ and Grim-like $\left(\mathrm{S}_{8}\right)$ rules which, as expected, can easily be overrun by TFT $\left(\mathrm{S}_{10}\right)$. However, large $\mathrm{e}$ favours the domination of defective strategies and the oscillations disappear.

In this model ALLD and Grim are the only ESSs, but the introduction of GTFT may lead to stable equilibria, where GTFT dominates (Nowak and Sigmund 1993b).

\section{Spatial chaos}

As already pointed out by Axelrod and Hamilton (1981), a cluster of unconditional cooperators can invade an ALLD population. Consider a population of players distributed on the squares of a chess board. Each player interacts only with its immediate 
neighbours, obeying the parameters of the singleshot Prisoner's Dilemma. In the next generation, the square is inhabited by the player who scored the highest total: neighbour or previous owner. Obviously, a single cooperator perishes, but just four cooperators in a cluster can get a foothold, since each interacts with more cooperators than a defector can reach. Nowak and May (1992) used cellular automata to simulate the spatial distribution of ALLD and ALLC 'territories' in a population. Surprisingly, their analysis of this simple deterministic model, with no memories among the contestants and without any strategical refinement, revealed spatial chaos: unpredictably everchanging spatial patterns of ALLD and ALLC neither vanishing.

In addition, Fig. 5 demonstrates that for certain parameters the frequencies are converging towards asymptotic fractions $f_{C}$ (and $f_{D}=1-f_{C}$, respectively). In spite of the stable relative frequencies, the chaotic fluctuations of the dynamic fractal last.

\section{Where is the Prisoner's Dilemma found in nature?}

In the last decade, the amount of game-theoretical literature on various aspects of the IPD has increased considerably. For most scientists the IPD has become something like a 'theoreticians' playground' with at times very little relation to empirical observations. Evidence of the mere existence of an IPD in biological societies is scarce, not only due to the difficulties in measuring the essential parameters but, as will be shown, also owing to the limited scope of the IPD. In this section I will try to relate

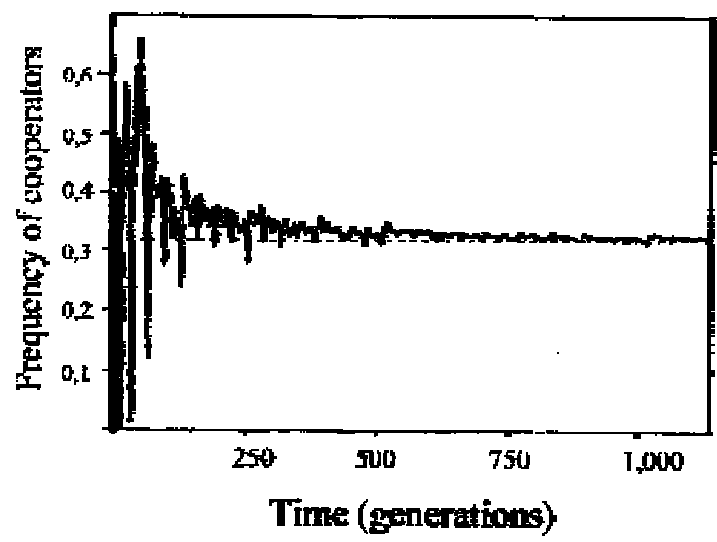

Fig. 5. The frequency of ALLC within the dynamic fractal generated by a single ALLD invading an ALLC population. (See Nowak and May 1992 for colour pictures of 'fractal kaleidoscooperation'). Redrawn from: Nowak, M. A. and May, R. M. 1992. Evolutionary games and spatial chaos. - Nature 359: 827. some empirical work from different taxa to the theoretical findings described so far.

\section{Myrmecophily}

One of the most recent attempts to investigate the structure underlying interspecific mutualism was performed by Leimar and Axén (1993). They studied the interaction of lycaenid butterfly larvae with ants. The caterpillars of many lycaenid species have a 'dorsal nectar organ', an exocrine gland that secretes a liquid containing carbohydrates and free amino acids. The ants harvest the liquid and in turn provide the larva with protection (see Pierce 1987 for more detailed information on the characteristics of this relationship). Since the sweet substance is costly to produce (Pierce 1987, Leimar and Axtén 1993), the larva would profit from obtaining ant attendance without releasing any liquid. On the other hand, ants may profit from harvesting the food rewards instead of defending the caterpillar. So both would do better defecting and thus $\mathrm{T}>\mathrm{R}>\mathrm{P}>\mathrm{S}$. The results from Leimar and Axén (1993) suggest that the caterpillars do respond to ant attendance, i.e. retaliate. Furthermore, $w$ might be sufficiently high, since the butterfly larvae never leave their host plants (Pierce 1987). However, there are several differences to the classical IPD: 1) The rewards to the players are asymmetrical. 2) The moves are not necessarily simultaneous. 3) The decision rules are influenced by a third party: predators and parasitoids attacking the larva (Leimar and Axtén 1993). 4) The investments in each interaction can be varied gradually (Leimar and Axén 1993). 5) Individual recognition is not likely between lycaenids and ants (Pierce 1987).

Hence the structure of lycaenid-ant interaction seems to resemble an IPD, but it is presumably more complex.

\section{Predator inspection in fish}

Probably one of the most often analysed phenomena in terms of the IPD, but also the most debated one is predator inspection in shoaling fish. When a predator is stalking a shoal of minnows (Phoxinus phoxinus), guppies (Poecilia reticulata) or sticklebacks (Gasterosteus aculeatus), individuals will separate from the shoal, swim tentatively towards the predator until only a few body lengths away, wait there for a few seconds and then slowly return to the shoal. It has been suggested that in such visits the fish can gather information about the identity, precise location and current motivational state of the predator (Milinski et al. 1990, Reboreda and Kacelnik 1990, Dugatkin and Alfieri 1991, Dugatkin 1991a, b, Turner and Robinson 1992). Two fish will approach the predator more closely than will single fish, so they both obtain the same benefit $b$ and share the cost $c(R=b-c / 2)$. If one fish lags behind the other, he will get the same 
information without the cost $(\mathrm{T}=\mathrm{b})$ while his sucker takes all the risks ( $\mathrm{S}=\mathrm{b}-\mathrm{c}$; Dugatkin and Alfieri 1992). If neither of them inspects, $c$ and $b$ are zero $(\mathrm{P}=0)$. If $\mathrm{c}>\mathrm{b}$ one condition for the Prisoner's Dilemma is satisfied: $\mathrm{T}>\mathrm{R}>\mathrm{P}>\mathrm{S}$ (Dugatkin and Alfieri 1991). Moreover, it is suggested that guppies (e.g. Dugatkin and Alfieri 1991) and sticklebacks (e.g. Milinski 1990) are capable of recognising previous defectors/cooperators, thus fulfilling another condition of the IPD.

Yet, there is no case study proving that all conditions are satisfied (Milinski 1992) and hence all experimental results are intensely debated (e.g. Milinski 1990, Lazarus and Metcalfe 1990, Reboreda and Kacelnik 1990, Dugatkin 1991b, Turner and Robinson 1992, Milinski 1992). An alternative way would be to assume the conditions for an IPD are fulfilled and predict the fishes' behaviour as specifically as possible. Assuming the fish use TFT, they must display the three characteristics associated with TFT: nicety, retaliation and forgiveness. Despite the problems translating these features into measurable, behavioural traits, Dugatkin (1991a) claims that his data support the use of TFT in guppies. His results indicate that guppies are 'nice' by beginning their initial inspection at about the same point in time, 'retaliatory' by turning back and making a fish that lagged to far behind (i.e. defected) the closest to the predator (i.e. defecting the defector) and 'forgiving' by sticking close to the former defector if he in turn now is approaching the predator. (See Dugatkin and Alfieri 1992 for similar results).

However, as already pointed out, the outcomes are debatable, since there is a lack of sufficient unambiguous evidence that fish do prefer to inspect in pairs (Turner and Robinson 1992). Moreover, if the knowledge gathered by inspecting fish is transferred to their non-inspecting shoalmates, as is commonly agreed upon, why should inspectors bear the cost of inspection but share the benefits (Dugatkin 1992)? How related are shoalmates with each other? Further experiments are needed to decide whether the IPD is an applicable model for predator inspection in fish.

\section{Egg-trading in simultaneous hermaphrodites}

Studying seabasses (Serranidae) may reduce measurements that help determine whether Axelrod's payoff matrix is satisfied. In these shallow water fishes, simultaneous hermaphroditism is a common feature (Fischer 1988, Conner 1992). They are known to be obligate outbreeders with external fertilisation and planktonic eggs. Such a system almost certainly prevents kin selection (Fischer 1988). During mating, each individual divides its clutch of eggs into parcels and, subsequent to courtship displays, alternates with its mate in offering parcels of eggs for fertilisation.
Owing to anisogamy, producing eggs is probably more costly than producing sperm. Thus the 'temptation to defect' i.e. fertilising eggs without giving any to be fertilised, is presumably high. Why do serranids still cooperate? Fischer (1988) suggested the fishes were using TFT and the system would apply to an IPD. However, there are several contradictions to this hypothesis: Fischer himself already pointed out (1988) that by dividing the clutches into a large number of parcels, the benefit $b$ (i.e. T) of fertilising a parcel of eggs is small. He also remarked that defection consists of the absence of an act. i.e. failure to offer eggs, rather than a specific behaviour. In his experiments (Fischer 1988) he referred to retaliation as waiting "significantly longer to release a batch of eggs than [...] if the partner did reciprocate". This is an obvious violation of the TFT rule. He concluded that the fish were playing a nicer version of TFT e.g. TF2T or GTFT. Yet, considering the evolution of the mating system, Connor (1992) proposed that an alternative explanation for cooperation in seabasses is more likely. It was suggested that simultaneous hermaphroditism evolved originally at a low density of conspecifics where this reproductive system has an advantage (Fischer 1988, Conner 1992). At that time the clutches were not parcelled. Changing ecological conditions increased the abundance of mates and parcelling appeared initially because it extended male mating success (Fischer 1988): a parcelling individual in a population of nonparcellers would therefore obtain more eggs from additional matings. Once parcelling became common, the seabasses found themselves in an IPD (Fischer 1988), possibly susceptible for a cheating strategy such as FR or Rover. Further decreasing each investment may ultimately have led to $R>T$ (Connor 1992) and thus the system escaped the IPD. This still remains to be tested.

If Conner's model applies, both individuals will cooperate until the last move, until there is only one parcel left. Not. offering this parcel will favour the withholding of the parcel on the penultimate move and so on. In Serranid seabasses, short spawning periods prevent cheating on the last move, as eggs must be spawned on the same day they are produced or they will become inviable. Since the chance of finding a new mate late in the spawning period is low, it is better to get its eggs fertilised than not to mate at all (Fischer 1988, Conner 1992). Moreover, Lima (1989) reported that cooperation may be stable in a finite IPD whose end point is known to all players.

\section{Blood-sharing in vampire bats}

Scientists working on cooperation among vampire bats (Desmondus rotundus) face the same difficulties in measuring all the essential parameters, but are in a somewhat better position. Some $8 \%$, of adult vampire bats fail to find food on any given 


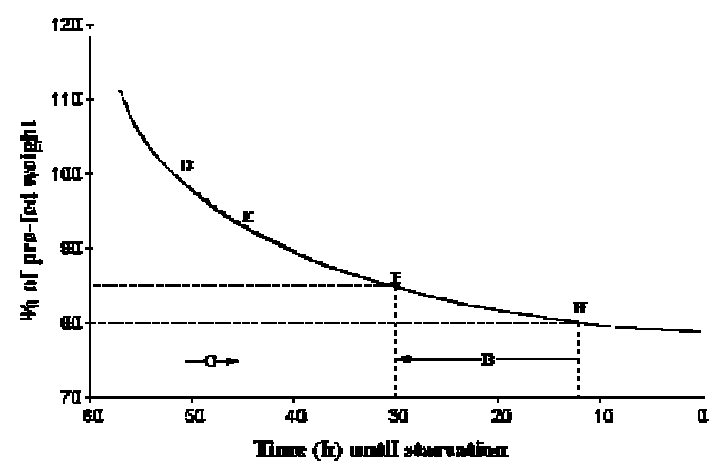

Fig. 6. Predictive curve of post feeding weightlosses in vampire bats (Desmondus rotundus). A donation of 5\% of pre-feeding weight when at weight $\mathrm{D}$ should cause a donor to lose $\mathrm{C}$ hours but provide the recipient at weight $\mathrm{H}$ with $\mathrm{B}$ hours. B $>\mathrm{C}$ for all $\mathrm{E}>\mathrm{F}$. See text for details. Redrawn from: Wilkinson, G. S. 1984. Reciprocal food sharing in the vampire bat. Nature 308: 183.

night (Wilkinson 1984), but on such nights without a blood meal they are often fed by successful roostmates. This behaviour is vital for the recipients, since their energy budget leaves them $48-72 \mathrm{~h}$ of food deprivation before starvation (McNab 1973). Wilkinson (1984) showed that food sharing by regurgitation of blood "depends equally and independently on degree of relatedness and an index of opportunity for reciprocation". He suggested that $\boldsymbol{w}$ is probably high enough to fit the IPD due to the long lifespan of the individuals and the stable composition of groups in which the average coefficient of relatedness is comparatively low (0.08-0.11; Wilkinson 1988). Fig. 6 shows that b>c because the exponential postfeeding weightless enables a donor to lose less time to starvation by regurgitation of blood than the recipient gains. Hence, it is plausible that $\mathrm{T}>\mathrm{R}>\mathrm{P}>\mathrm{S}$. Furthermore, the cognitive abilities of vampire bats appear to be sufficiently well-developed to guarantee recognition of cooperators and defectors (Wilkinson 1984). While it is reported that bats do in fact deny feeding those roostmates that refused to regurgitate previously (i.e. act retaliatorily; Wilkinson 1984), it still remains to find out which strategy the bats actually use. An applicability of the IPD should be confirmed by further investigations.

\section{Reciprocal altruism in primates}

While struggling to understand the evolution of cooperation throughout the different taxa, one of the most intricate tasks is to study cooperation in primates. Packer (1977) was the first to report reciprocal altruism among male olive baboons (Papio anuhis). The formation of short-term coalitions among adult male $P$. anuhis during aggressive interaction against a single adversary is initiated by one male enlisting a partner and completed by subsequently fighting the opponent.
Packer found considerable evidence that individuals who join an enlisting baboon have previously succeeded in enlisting that very same individual, suggesting cooperation may be partly based on reciprocity (Packer 1977). Determining costs and benefits is extremely difficult, since the payoff matrix may vary according to the different circumstances under which coalitions are formed (e.g. the strength of the opponent specifies the costs, while the cause of the aggression specifies the benefits). However, assuming that most of the coalitions were formed to take over an oestrous female and that the wounds received were non-fatal, the resulting sired offspring would be a greater benefit than the less costly wounds received in a number of consecutive 'plays' if both partners reciprocated in aiding each other (Packer 1977).

But since only one of the cooperating partners (usually the enlisting male) obtains the female at issue, two coalitions make a 'game'. This case is at best the alternating variant of the simultaneous IPD, recently investigated by Nowak and Sigmund (1994). Noë (1990) argued that this form of delayed reciprocity is better explained with a 'Veto game' that includes information exchange, partner-choice and partner competition. The ability of primates in trading different beneficial acts for other behaviour supporting the benefactor, further complicates the matter. Seyfarth and Cheney (1984) looked at coalition-forming in female vervet monkeys (Cercopithecus aethiops) in relation to preceded grooming. Their data seem to support the view that vervets are more apt to help an enlisting, unrelated individual if they have been groomed by those individuals in the recent past (Seyfarth and Cheney 1984). However, due to the complexity of exchanging acts in various currencies (especially if more than two behaviours are involved) and due to the difference in hierarchical rank that breaks the symmetry of the game, quantification of costs and benefits seems unlikely to be possible (Noë 1990). Therefore, all results obtained in studies as the two described above will be persistently disputed (e.g. Noë 1990, Hemelrijk 1991, Seyfarth 1991).

\section{Conclusion}

Cooperation is found in most taxa. One has to keep in mind, that cooperation never was a goal but rather a better solution than others to problems that undoubtedly occurred frequently during the evolution of life. Why?

On the foregoing pages I tried to draw a short sketch of the current state of research on the evolution of cooperation. As we have seen in the first four sections, this interest in the evolution of cooperation has led to independent theoretical work on the IPD for its own sake. An outstanding performance of 
theorists in the last decade has provided us with a large number and diversity of papers investigating or elaborating theoretical concepts within the IPDframework. Nevertheless, "our understanding of the evolution of cooperation is at such an elementary stage as to suggest that additional paradigms remain to be developed" (Bull and Rice 1991). That hitherto no biological system could be found in which all the conditions for an IPD were met, clearly demands new approaches, which will be more readily testable. In more general models, random mutations might provide its bearers with traits that could change environmental, physiological and developmental parameters. Environments, selection pressures or mutation rates are neither fixed nor imposed, inaccessible to evolution, but rather highly dynamic variables. I agree with Dugatkin et al. (1992) and Mesterton-Gibbons and Dugatkin (1992) that "it is time for the theoretical work to go beyond the [...] IPD" towards paradigms that include reciprocal altruism, by-product mutualism and other potential categories of cooperation between nonrelatives. The aim, of course, is to combine such new paradigms to a model, that would provide a powerful tool to investigate, under which precise conditions, which forms of cooperation could evolve.

Assume that an observed behaviour corresponds to one solution of the game. Then, one can try to find the conditions under which this observed behaviour is evolutionarily stable. In this context, the stability question can be put at several levels: the stability of single strategies (Lorberbaum 1994), the stability of relative frequencies in an ensemble of strategies (Feldmann and Thomas 1987), the stability of oscillations in these frequencies (Nowak and Sigmund 1993b), etc. The construction of such a general model would eliminate several weaknesses of the IPD as well (Dugatkin et al. 1992, MestertonGibbons and Dugatkin 1992): 1) It would allow the exchange of information between the interactants. Only few higher species ignore signals (of willingness to cooperate or threat to break the partnership) from their opponents during interaction (see Noë 1990 for a model including information exchange). 2) It would allow to change the geometric probability distribution of $\Theta$ from $\operatorname{Prob}(\Theta)=w^{\Theta-1}$ to a behaviour dependent distribution. The formation of preference between successful partners will certainly flatten the steep decline of $\operatorname{Prob}(\Theta)$ with $\Theta$ (see Feldmann and Thomas 1987, Noë 1990). 3) While the IPD models the maintenance of cooperation fairly well, it still fails to elucidate the origin of cooperation from an asocial state (see Feldmann and Thomas 1987). 4) In the IPD, there are only two choices: C or D. Corresponding punishment of different nuances of defection seems more realistic (see Clutton-Brock and Parker 1995 for the role of punishment, and Leimar and Axén 1993 for a discussion on gradually reactive behaviour). 5) A new model would enlarge the scope to N-player games (see Dugatkin 1990, Noë 1990) in order to model the evolution of social groups.

However, evolution is a historical process (see also Quenette and Gerard 1993) acting on a dynamical landscape, producing unique solutions to each new problem. Simulations of evolution should also be expected to have different outcomes for every run.

Acknowledgements - I would like to thank Göran Englund, Martin Nowak, Neil Metcalfe, Chris Cutts, Alfredo Nicieza and Martin Heisenberg for their support and encouragement.

\section{References}

Axelrod, R. and Hamilton, W. D. 1981. The evolution of cooperation. - Science 211: 1390-1396.

Bendor, J. and Swistak, P. 1995. Types of evolutionary stability and the problem of cooperation. - Proc. Natl. Acad. Sci. 92: 3596-3600.

Boyd, R. and Lorberbaum, J. P. 1987. No pure strategy is evolutionary stable in the repeated prisoner's dilemma game. Nature 327: 58-59.

Bull, J. J. and Rice, W. R. 1987. Distinguishing mechanisms for the evolution of cooperation. - J. theor. Biol. 149: 63-74.

Clutton-Brock, T.H. and Parker, G. A. 1995. Punishment in animal societies. - Nature 373: 209216.

Connor, R. C. 1992. Egg-trading in simultaneous hermaphrodites: an alternative to tit-for-tat. - J. evol. Biol. 5: 523-528.

Darwin, C. 1859. The Origin of Species, Penguin Books, London.

Dugatkin, L. A. 1990. N-person games and the evolution of co-operation: a model based on predator inspection in fish. - J. theor. Biol. 142: 123-135.

Dugatkin, L. A. 1991a. Dynamics of the tit for tat strategy during predator inspection in the guppy (Poecilia reticulata). - Behav. Ecol. Sociobiol. 29: 127-132.

Dugatkin, L. A. 1991b. Predator inspection, tit-for-tat and shoaling: a comment on Masters \& Waite. - Anim. Behav. 41: 898-899.

Dugatkin, L. A. 1992. Tendency to inspect predators predicts mortality risk in the guppy (Poecilia reticulata). - Behav. Ecol. 3: 124-127.

Dugatkin, L. A. and Alfieri, M. 1991. Guppies and the tit for tat strategy: preference based on past interaction. - Behav. Ecol. Sociobiol. 28: 243-246.

Dugatkin, L. A. and Alfieri, M. 1992. Interpopulational differences in the use of the tit-for-tat strategy during predator inspection in the guppy, Poecilia reticulata. - Evol. Ecol. 6: 519-526.

Dugatkin, L. A. , Mesterton-Gibbons, M. and Houston, A. I. 1992. Beyond the prisoner's dilemma: toward models to discriminate among mechanisms of cooperation in nature. - Trends. Ecol. Evol. 7: 202205.

Dugatkin, L. A. and Wilson, D. 1991. Rover: a strategy for exploiting cooperators in a patchy environment. - Am. Nat. 138: 687-701. 
Eldredge, N. and Gould, S. J. 1972. In: Schopf, T. J. M. (ed.), Models in paleobiology. - Freeman, Cooper, San Francisco: 82-115.

Enquist, M. and Leimar, O. 1993. The evolution of cooperation in mobile organisms. - Anim. Behav. 45: 747-757.

Feldmann, M. W. and Thomas, A. C. 1987. Behaviour dependent contexts for repeated plays of the prisoner's dilemma. II. Dynamical aspects of the evolution of cooperation. - J. theor. Biol. 128: 297315.

Fischer, E. A. 1988. Simultaneous hermaphroditism, titfor-tat, and the evolutionary stability of social systems. - Ethol. Sociobiol. 9: 119-136.

Gould, S. J. and Eldredge, N. 1993. Punctuated equilibrium comes of age. - Nature 366: 223-227.

Hamilton, W. D. 1964. The genetical evolution of social behavior. - J. theor. Biol. 7: 1-32.

Hemelrijk, C. K. 1991. Interchange of "altruistic" acts as an epiphenomenon. - J. theor. Biol. 153: 137-139.

Lazarus, J and Metcalfe, N. B. 1990. Tit-for-tat cooperation in sticklebacks: a critique of Milinski. - Anim. Behav. 30: 987-988.

Leimar, O. and Axén, A. H. 1993. Strategic behavior in an interspecific mutualism: interactions between lycaenid larvae and ants. - Anim. Behav. 46: 11771182.

Lima, S. L. 1989. Iterated prisoner's dilemma: an approach to evolutionary stable cooperation. - Am. Nat. 134: 828-834.

Lorberbaum, J. 1994. No strategy is evolutionary stable in the repeated prisoner's dilemma. - J. theor. Biol. 168: $117-130$.

Maynard Smith, J. 1982. Evolution and the theory of games. - Cambridge University Press, UK.

McNab, B. K. 1973. Energetics and the distribution of vampires. - J. Mammal. 54: 131-144.

Mesterton-Gibbons, M. 1991. An escape from 'the prisoner's dilemma'. - J. Math. Biol. 29: 251-269.

Mesterton-Gibbons, M. and Dugatkin, L. A. 1992. Cooperation among unrelated individuals: evolutionary factors. - Q. Rev. Biol 67: 267-281.

Milinski, M. 1990. On cooperation in sticklebacks. Anim. Behav. 40: 1190-1191.

Milinski, M. 1992. Predator inspection: cooperation or 'safety in numbers'? - Anim. Behav. 43: 679-680.

Milinski, M. , Külling, D. and Kettler, R. 1990. Tit for Tat: sticklebacks (Gasterosteus aculeatus) "trusting" a cooperating partner. - Behav. Ecol. 1: 7-11.
Noë, R. 1990. A veto game played by baboons: a challenge to the use of the prisoner's dilemma as a paradigm for reciprocity and cooperation. - Anim. Behav. 39: 78-90.

Nowak, M. A. and May, R. M. 1992. Evolutionary games and spatial chaos. - Nature 359: 826-829.

Nowak, M. A. and Sigmund, K. 1992. Tit for tat in heterogeneous populations. - Nature 355: 250-253.

Nowak, M. A. and Sigmund, K. 1993a. Chaos and the evolution of cooperation. - Proc. Natl. Acad. Sci. USA 90: 5091-5094.

Nowak, M. A. and Sigmund, K. 1993b. A strategy of win-stay lose-shift that outperforms tit-for-tat in the Prisoner's Dilemma game. - Nature 364: 56-58.

Nowak, M. A. and Sigmund, K. 1994. The alternating prisoner's dilemma. - J. theor. Biol. 168: 219-226.

Packer, C. 1977. Reciprocal altruism in Papio anubis. Nature 265: 441-443.

Pierce, N. E. 1987. The evolution and biogeography of associations between lycaenid butterflies and ants. - Oxf. Surv. Evol. Biol. 4: 89-116.

Pollock, G. and Dugatkin, L. A. . 1992. Reciprocity and the emergence of reputation. - J. theor. Biol. 159: 25-37.

Quenette, P. Y. and Gerard, J. F. 1993. Why biologists do not think like Newtonian physicists. - Oikos 68 : 361-363.

Reboreda, J. C. and Kacelnik, A. 1990. On cooperation, tit-for-tat and mirrors. - Anim. Behav. 40: 11881189.

Seyfarth, R. M. 1991. Reciprocal altruism and the limits of correlational analysis. - J. theor. Biol. 153: 141144.

Seyfarth, R. M. and Cheney, D. L. 1984. Grooming, alliances and reciprocal altruism in vervet monkeys. - Nature 308: 541-543.

Trivers, R. L. 1971. The evolution of reciprocal altruism. - Q. Rev. Biol. 46: 35-57.

Turner, G. F. and Robinson, R. L. 1992. Milinski's titfor-tat hypothesis: do fish preferentially inspect in pairs? - Anim. Behav. 43: 677-678.

Wilkinson, G. S. 1984. Reciprocal food sharing in the vampire bat. - Nature 308: 181-184.

Wilkinson, G. S. 1988. Reciprocal altruism in bats and other mammals. - Ethol. Sociobiol. 9: 85-100. 